\title{
A BANACH SPACE SOLUTION OF AN ABSTRACT CAUCHY PROBLEM WHICH HAS ROBIN BOUNDARY DATA
}

\author{
BY \\ DENNIS W. QUINN \\ Air Force Institute of Technology, Wright-Patterson Air Force Base, Ohio
}

\begin{abstract}
In this paper, a problem which involves the diffusion equation coupled by way of Robin boundary data with a nonlinear system of ordinary differential equations is considered. This problem has been proposed as a model to describe the absorption through the skin, the distribution throughout the body, and the metabolism of a substance in a mammal. The problem is set as an abstract Cauchy problem in a Banach space and is shown to have a unique solution. Finite dimensional approximations of the problem are obtained by replacing the spatial partial derivatives with finite differences. The approximate solutions are shown to converge to the exact solution of the original problem. Comparisons of numerical solutions with experimental data are presented.
\end{abstract}

I. Introduction. It is well known that many complicated phenomena can be adequately described by a system of ordinary differential equations. On the other hand, many phenomena are adequately modeled only if we use partial differential equations. In this paper we consider problems which can be described by a system of ordinary differential equations which is coupled to a partial differential equation. In particular, we consider processes which include diffusion coupled with mass-balance transport. An example of such a process is the absorption through the skin, and then the transport and metabolism of substances in a mammal. In [1], experimental data has recently been obtained for skin (dermal) exposure to two dihalomethanes. In [2], the absorption through the skin, the distribution throughout the body of the mammal, and the metabolism into carbon dioxide and carbon monoxide for dihalomethanes have been described as a nonlinear system of ordinary differential equations. The description in [2] assumes that each tissue group within the mammal, including the skin, can be viewed as a compartment. The movement of the dihalomethanes between compartments is described by mass-balance equations which result in the differential equations of the system.

In this paper we address the problem in [1] by having the diffusion equation coupled with a system of five mass-balance ordinary differential equations, which describe the distribution and metabolism. In Sec. II, these equations are presented and 
the problem of solving the system of equations is formulated as an abstract Cauchy problem (an evolution equation in a Banach space). The boundary conditions for the diffusion equation dictate the way that the equations are coupled and are important in the analysis. The diffusion equation has been considered in the context of an abstract Cauchy problem in references [3], [4], [5], [6], [7], and [8] for Dirichlet (in which the function is specified) and Neumann (in which the derivative of the function is specified) boundary data and in [3] and [7] for Robin boundary data (in which a linear combination of the function and its derivative is specified). In this paper, Robin boundary data is a key feature. In [3], Banks and Kunisch present a general framework for approximation schemes for abstract Cauchy problems. Reference [3] has been a guide for several of our results.

In Sect. III, the existence of a unique solution of our abstract Cauchy problem is shown to follow from the Hille-Yosida theorem. In Sect. IV, approximate problems are obtained when spatial derivatives in the diffusion equation are replaced by finite differences. This results in a sequence of semigroups in a sequence of Banach spaces which is consistent with the Trotter-Kato theorem. In Sect. V, numerical results are discussed, including a comparison of theoretical predictions and experimental observations in [1].

II. Equations and formulation as evolution equation. In [2], a physiological model for dermal vapor absorption is presented based on data collected in [1]. The compartmental description which was used in [2] is similar to the diagram in Fig. 1. Reference [2] includes the following four mass-balance equations to relate $C_{F}, C_{S}, C_{R}, C_{L}$, and $C_{A}$, which are respectively the concentrations of dibromomethane in the fat, slowly perfused tissue, rapidly perfused tissue, liver, and arterial blood.

$$
\begin{aligned}
V_{F} \frac{d C_{F}}{d t} & =Q_{F}\left(C_{A}-\frac{C_{F}}{P_{F}}\right), \\
V_{S} \frac{d C_{S}}{d t} & =Q_{S}\left(C_{A}-\frac{C_{S}}{P_{S}}\right), \\
V_{R} \frac{d C_{R}}{d t} & =Q_{R}\left(C_{A}-\frac{C_{R}}{P_{R}}\right), \\
V_{L} \frac{d C_{L}}{d t} & =Q_{L}\left(C_{A}-\frac{C_{L}}{P_{L}}\right)-\frac{V_{M} C_{L}}{K_{M} P_{L}+C_{L}}-\frac{K_{F} C_{L} V_{L}}{P_{L}} .
\end{aligned}
$$

Physiologically, the quantities $P_{F}, P_{S}, P_{R}$, and $P_{L}$ are tissue to blood partition coefficients for, respectively, the fat, slowly perfused tissue, rapidly perfused tissue, and liver; $Q_{F}, Q_{S}, Q_{R}$, and $Q_{L}$ are, respectively, the blood flow rates in the fat, slowly perfused tissues, rapidly perfused tissues, and liver; $V_{F}, V_{R}, V_{S}$, and $V_{L}$ are respectively the volumes of fat, rapidly perfused tissue, slowly perfused tissue, and liver; $V_{M}$ is the maximum specific reaction velocity for the dihalomethane; $K_{M}$ is the Michaelis constant; and $K_{F}$ is the first-order rate constant. Note that the nonlinear term in Eq. (2.1.d) models the effects of metabolism in the liver.

The arterial blood concentration $C_{A}$ is calculated using the equation $C_{A}=$ $Q_{C} C_{V} /\left(Q_{P} / P_{B}+Q_{C}\right)$, where $C_{V}$ is the venous blood concentration and is 


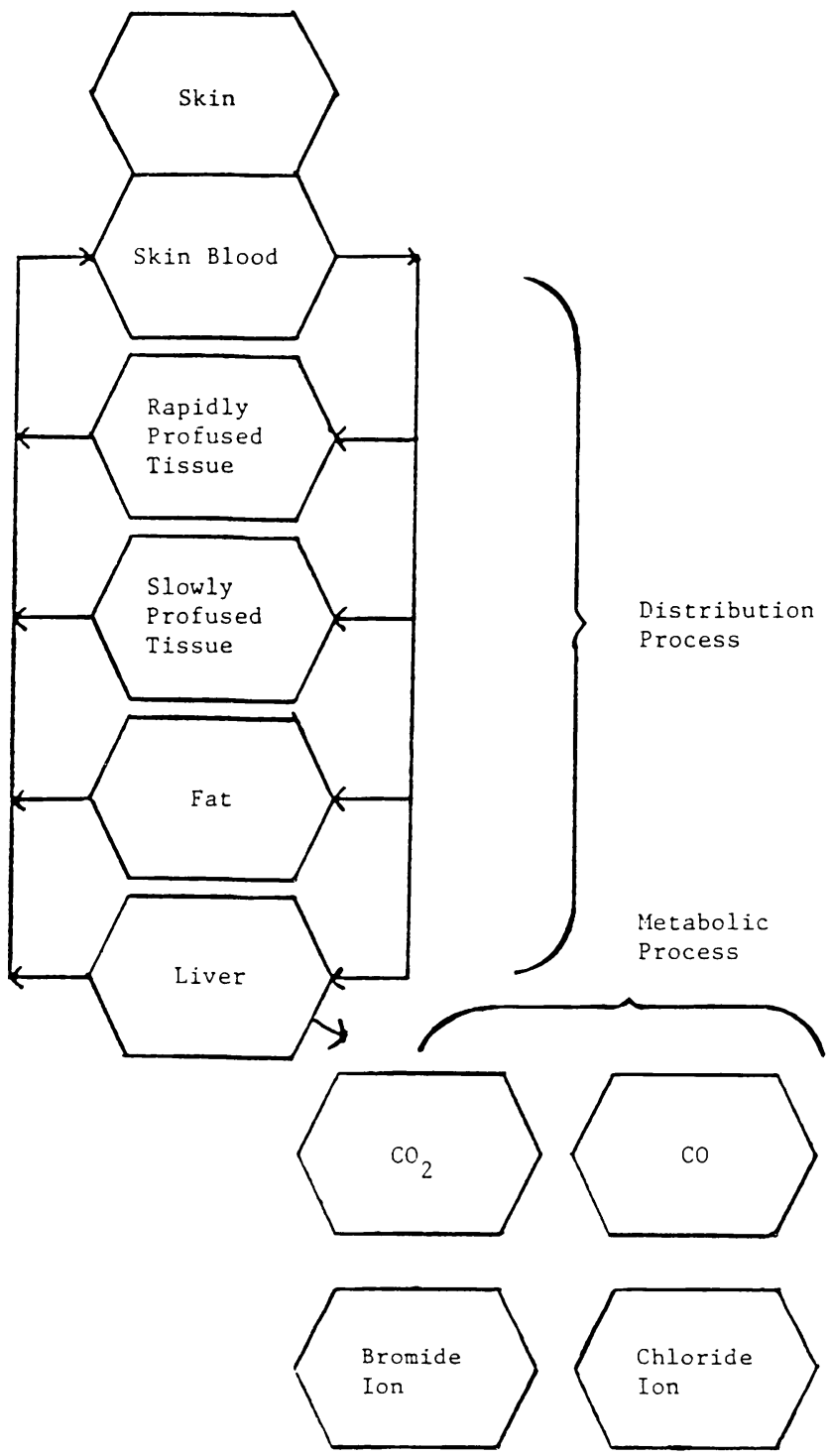

Fig. 1. Compartmental model.

determined by

$$
C_{V}=\left[Q_{F} \frac{C_{F}}{P_{F}}+Q_{S} \frac{C_{S}}{P_{S}}+Q_{R} \frac{C_{R}}{P_{R}}+Q_{L} \frac{C_{L}}{P_{L}}+Q_{S K} \frac{C_{S K}}{P_{S K}}\right] / Q_{C} ;
$$

$Q_{C}$ and $Q_{P}$ are the cardiac and pulmonary output; $P_{S K}$ and $P_{B}$ are skin to air and blood to air partition coefficients; $Q_{S K}$ is the blood flow rate in the skin; and $C_{S K}$ is the concentration of dihalomethane in the skin.

In [2], the skin of the mammal was viewed as a compartment for which a massbalance equation of the form of Eqs. (2.1.a)-(2.1.d) was appropriate. The equation 
which was used in [2] is

$$
V_{S K} \frac{d C_{S K}}{d t}=P A\left(C_{0}-\frac{C_{S K}}{P_{S K}}\right)+Q_{S K}\left(C_{A}-\frac{C_{S K}}{P_{S K B}}\right) .
$$

In Eq. (2.1.e), $C_{0}$ is the concentration of dihalomethane at the outer surface of the skin; $P_{S K B}$ is the tissue to blood partition coefficient for the skin; $V_{S K}$ is the volume of skin; $P$ is the permeability of the skin; and $A$ is the area of skin exposed to the dihalomethane.

Note that Eqs. (2.1.a)-(2.1.e) comprise a coupled system of five ordinary differential equations and that $C_{A}$ depends on each of the other concentrations and that the presence of the $C_{A}$ term in each equation results in each concentration depending on each of the other concentrations.

To more accurately describe the diffusion of dibromomethane through the skin, we replaced Eq. (2.1.e) with the diffusion equation, a partial differential equation, and added an equation to describe the concentration in the skin blood. This yields the following system

where

(b) $V_{S B} \frac{d C_{S B}}{d t}=P_{B C} A\left(\frac{u(L, t)}{P_{S K B}}-C_{S B}\right)+Q_{S K}\left(C_{A}-C_{S B}\right)$,

(c) $\quad V_{F} \frac{d C_{F}}{d t}=Q_{F}\left(C_{A}-\frac{C_{F}}{P_{F}}\right)$,

(d) $\quad V_{S} \frac{d C_{S}}{d t}=Q_{S}\left(C_{A}-\frac{C_{S}}{P_{S}}\right)$,

(e) $\quad V_{R} \frac{d C_{R}}{d t}=Q_{R}\left(C_{A}-\frac{C_{R}}{P_{R}}\right)$,

(f) $V_{L} \frac{d C_{L}}{d t}=Q_{L}\left(C_{A}-\frac{C_{L}}{P_{L}}\right)-\frac{V_{M} C_{L}}{K_{M} P_{L}+C_{L}}-\frac{K_{F} C_{L} V_{L}}{P_{L}}$,

and

$$
C_{A}=\frac{Q_{C} C_{V}}{Q_{P} / P_{B}+Q_{C}}
$$

$$
C_{V}=\left[Q_{F} \frac{C_{F}}{P_{F}}+Q_{S} \frac{C_{S}}{P_{S}}+Q_{R} \frac{C_{R}}{P_{R}}+Q_{L} \frac{C_{L}}{P_{L}}+Q_{S K} \frac{C_{S K}}{P_{S K}}\right] / Q_{C} .
$$

Because Eq. (2.2.a) is a partial differential equation of parabolic type, the following boundary conditions have been added to the model

$$
\begin{aligned}
& u_{x}(0, t)=h_{1}\left[u(0, t)-P_{S K} C_{0}\right], \\
& u_{x}(L, t)=h_{2}\left[P_{S K B} C_{S B}-u(L, t)\right] .
\end{aligned}
$$

These conditions state that the flux of concentration across the skin boundary at both the skin-air interface and the skin-blood interface is proportional to the difference of the concentration in the skin at the interface and the concentration on the skin at the interface, taking into account the partition coefficients $P_{S K}$ and $P_{S K B}$. 
The initial condition for $u$ is

$$
u(x, 0)=0 .
$$

In the system of equations, $u, C_{S K}, C_{F}, C_{S}, C_{R}, C_{L}, C_{0}, C_{V}$, and $C_{A}$ are functions of time. All other quantities are constants. The values of these constants are displayed in Table 1. For the four experiments in [1], the values for $C_{0}$ were $.00356, .00711, .0356$, and $.0711 \mathrm{mg} / \mathrm{ml}$, respectively, while the values for $B_{W}$, the body weight, were $.187, .212, .209$, and $.260 \mathrm{~kg}$, respectively. In Table 1, once the body weight is specified, all the other constants can be calculated for inclusion in Eqs. (2.1) or (2.2).

TABLE 1

\begin{tabular}{cccc} 
CONSTANT & VALUE & CONSTANT & VALUE \\
\hline$Q_{C}$ & $15 B_{W}^{.74}$ & $A$ & $9.1\left(1000 . B_{W}\right)^{.666}-60$ \\
$Q_{F}$ & $.090 Q_{C}$ & $V_{F}$ & $.07 B_{W}$ \\
$Q_{R}$ & $.560 Q_{C}$ & $V_{R}$ & $.05 B_{W}$ \\
$Q_{L}$ & $.200 Q_{C}$ & $V_{L}$ & $.04 B_{W}$ \\
$Q_{S K}$ & $.05 A Q_{C} /\left(.906 B_{W}^{.7}\right)$ & $V_{S K}$ & $.01 A$ \\
$Q_{S}$ & $.15 Q_{C}-Q_{S K} / 1000$. & $V_{S}$ & $.75 B_{W}$ \\
$Q_{P}$ & $15 B_{W}^{.74}$ & $V_{M}$ & $10.6 B_{W}^{.7}$ \\
$K_{F}$ & $.557 / B_{W}^{.3}$ & $K_{M}$ & .360 \\
$P_{B}$ & 74.1 & $P_{S K}$ & 266. \\
$P_{S K B}$ & $P_{S K} / P_{B}$ & $P$ & 1.100 \\
$P_{F}$ & 10.800 & $P_{R}$ & .900 \\
$P_{L}$ & .919 & $P_{S}$ & .546 \\
$k$ & $.01 P$ & $P_{B C}$ & .34 \\
$h_{1}$ & .27 & $h_{2}$ & 5.5
\end{tabular}

If the expression for $C_{V}$ is substituted into the expression for $C_{A}$ and this in turn is substituted inio Eqs. (2.2.b) through (2.2.f), we obtain the following system of equations

(a) $\quad u_{t}=k u_{x x} \quad 0<x<L$,

(b) $d C_{S B} / d t=B_{21} u(L, t)+B_{22} C_{S B}+B_{23} C_{F}+B_{24} C_{S}+B_{25} C_{R}+C_{26} C_{L}$,

(c) $d C_{F} / d t=\quad B_{32} C_{S B}+B_{33} C_{F}+B_{34} C_{S}+B_{35} C_{R}+C_{36} C_{L}$,

(d) $d C_{S} / d t=\quad B_{42} C_{S B}+B_{43} C_{F}+B_{44} C_{S}+B_{45} C_{R}+C_{46} C_{L}$,

(e) $d C_{R} / d t=\quad B_{52} C_{S B}+B_{53} C_{F}+B_{54} C_{S}+B_{55} C_{R}+C_{56} C_{L}$,

(f) $d C_{L} / d t=\quad B_{62} C_{S B}+B_{63} C_{F}+B_{64} C_{S}+B_{65} C_{R}+C_{66} C_{L}$, $+N_{1} C_{L} /\left(N_{2}+C_{L}\right)$

where $B_{21}=P_{B C} A /\left(V_{S B} P_{S K B}\right), \quad B_{22}=T_{2} Q_{S K}-Q_{S K} / V_{S B}-P_{B C} A / V_{S B}, B_{23}=$ $T_{2} Q_{F} / P_{F}, \quad B_{24}=T_{2} Q_{S} / P_{S}, \quad B_{25}=T_{2} Q_{R} / P_{R}, \quad B_{26}=T_{2} Q_{L} / P_{L}, \quad$ and 


$$
\begin{aligned}
& T_{2}=Q_{S K} T_{1} / V_{S B} \text { with } T_{1}=1 /\left(Q_{P} / P_{B}+Q_{C}\right) \text {. Similarly, } \\
& B_{32}=T_{3} Q_{S K}, \quad B_{33}=T_{3} Q_{F} / P_{F}-Q_{F} /\left(P_{F} V_{F}\right), \\
& B_{34}=T_{3} Q_{S} / P_{S}, \quad B_{35}=T_{3} Q_{R} / P_{R}, \\
& B_{36}=T_{3} Q_{L} / P_{L} \quad \text { and } T_{3}=Q_{F} T_{1} / V_{F} ; \\
& B_{42}=T_{4} Q_{S K}, \quad B_{43}=T_{4} Q_{F} / P_{F}, \\
& B_{44}=T_{4} Q_{S} / P_{S}-Q_{S} /\left(P_{S} V_{S}\right), \quad B_{45}=T_{4} Q_{R} / P_{R}, \\
& B_{46}=T_{4} Q_{L} / P_{L} \quad \text { with } T_{4}=Q_{S} T_{1} / V_{S} ; \\
& B_{51}=T_{5} Q_{S K}, \quad B_{52}=T_{5} Q_{F} / P_{F}, \\
& B_{54}=T_{5} Q_{S} / P_{S}, \quad B_{55}=T_{5} Q_{R} / P_{R}-Q_{R} /\left(P_{R} V_{R}\right), \\
& B_{56}=T_{5} Q_{L} / P_{L} \quad \text { with } T_{5}=Q_{R} T_{1} / V_{R} ; \\
& B_{62}=T_{6} Q_{S K}, \quad B_{63}=T_{6} Q_{F} / P_{F}, \\
& B_{64}=T_{6} Q_{S} / P_{S}, \quad B_{65}=T_{6} Q_{R} / P_{R}, \\
& B_{66}=T_{6} Q_{L} / P_{L}-Q_{L} /\left(P_{L} V_{L}\right)-K_{F} / P_{L} \quad \text { with } T_{6}=Q_{L} T_{1} / V_{L} ; \\
& N_{1}=-V_{M} / V_{L} \text { and } N_{2}=K_{M} P_{L} .
\end{aligned}
$$

The concentrations $C_{S B}, C_{F}, C_{S}, C_{R}$, and $C_{L}$ are all zero initially. The quantities $k, B_{21}, \ldots, B_{66}, N_{1}, N_{2}, h_{1}, P_{S K}, P_{S K B}$ and $h_{2}$ are all constants in this analysis and are described in terms of physiological quantities such as organ volumes, blood flow rates and partition coefficients. The constants $N_{2}, k, h_{1}, P_{S K}, P_{S K B}$, and $h_{2}$ are positive.

The boundary conditions can be transformed into homogeneous boundary conditions by introducing the function

$$
\begin{aligned}
w(x, t)= & \left(1-h_{2} L-h_{2} x\right)\left(h_{1} P_{S K} C_{0}\right) /\left(h_{1}+h_{2}+h_{1} h_{2} L\right) \\
& +\left(h_{1} x+1\right) h_{2} P_{S K B} C_{S B} /\left(h_{1}+h_{2}+h_{1} h_{2} L\right)
\end{aligned}
$$

and letting

$$
u_{1}(x, t)=u(x, t)-w(x, t) .
$$

Then $u_{1}(x, t)$ satisfies the boundary conditions

$$
\begin{aligned}
& u_{1 x}(0, t)=h_{1} u(0, t), \\
& u_{1 x}(L, t)=-h_{2} u(L, t),
\end{aligned}
$$

and the initial condition

$$
u_{1}(x, 0)=\left(h_{2} x-1-h_{2} L\right)\left(h_{1} P_{S K} C_{0}\right) /\left(h_{1}+h_{2}+h_{1} h_{2} L\right) .
$$

In this analysis, $C_{0}$ does not change with time, and consequently, the partial differential equation which $u_{1}$ satisfies is

$$
u_{1 t}=k u_{1 x x}+(m x+b) d C_{S B} / d t
$$

where

$$
m=-h_{1} h_{2} P_{S K B} /\left(h_{1}+h_{2}+h_{1} h_{2} L\right)
$$

and

$$
b=-h_{2} P_{S K B} /\left(h_{1}+h_{2}+h_{1} h_{2} L\right) .
$$


The $d C_{S B} / d t$ term is eliminated from Eq. (2.7) by replacing it with the right-hand side of Eq. (2.4b). If the vector function $U(t)=\left(u_{1}(x, t), u_{2}(t), \ldots, u_{6}(t)\right)^{T}$ is introduced with $u_{2}(t)=C_{S B}, u_{3}(t)=C_{F}, u_{4}(t)=C_{S}, u_{5}(t)=C_{R}$, and $u_{6}(t)=C_{L}$, and $\varphi(x)=m x+b$, then system $(2.1)$ can be written as

where

$$
d U / d t=A U+B U+F(U)
$$

$$
A=\left[\begin{array}{cccccc}
k(\cdot)_{x x} & 0 & 0 & 0 & 0 & 0 \\
0 & -1 & 0 & 0 & 0 & 0 \\
0 & 0 & -1 & 0 & 0 & 0 \\
0 & 0 & 0 & -1 & 0 & 0 \\
0 & 0 & 0 & 0 & -1 & 0 \\
0 & 0 & 0 & 0 & 0 & -1
\end{array}\right]
$$

$$
\begin{gathered}
B=\left[\begin{array}{cccccc}
\varphi(x) B_{21} S_{L} & \varphi(x) B_{22} & \varphi(x) B_{23} & \varphi(x) B_{24} & \varphi(x) B_{25} & \varphi(x) B_{26} \\
B_{21} S_{L} & B_{22}+1 & B_{23} & B_{24} & B_{25} & B_{26} \\
0 & B_{32} & B_{33}+1 & B_{34} & B_{35} & B_{36} \\
0 & B_{42} & B_{43} & B_{44}+1 & B_{45} & B_{46} \\
0 & B_{52} & B_{53} & B_{54} & B_{55}+1 & B_{56} \\
0 & B_{62} & B_{63} & B_{64} & B_{65} & B_{66+1}
\end{array}\right] \\
F(U)=\left[\begin{array}{c}
\varphi(x) B_{21}\left(B_{F 1}+B_{F 2} u_{2}\right) \\
B_{21}\left(B_{F 1}+B_{F 2} u_{2}\right) \\
0 \\
0 \\
0 \\
N_{1} u_{6} /\left(N_{2}+u_{6}\right)
\end{array}\right] \\
B_{F 1}=\left(1-2 h_{2} L\right)\left(h_{1} P_{S K} C_{0}\right) /\left(h_{1}+h_{2}+h_{1} h_{2} L\right), \\
B_{F 2}=\left(h_{1} L+1\right) h_{2} P_{S K B} u_{2} /\left(h_{1}+h_{2}+h_{1} h_{2} L\right),
\end{gathered}
$$

and the operator $S_{L}$ is defined by

$$
S_{L} u_{1}(x, t)=u_{1}(L, t) .
$$

The initial condition for $U$ is

$$
U(0)=U_{0}=(-w(x, 0), 0,0,0,0,0,)^{T} .
$$

The function space $D(A)$ on which solutions to $(2.8)$ will be sought is defined as follows:

DEFINITION 2.10.

a. $P=\left\{f \in C^{\infty}[0, L] \times R^{5}: d f_{1} / d x-h_{1} f_{1}=0\right.$ outside some compact subset of $(0, L]$ and $d f_{1} / d x+h_{2} f_{1}=0$ outside some compact subset of $\left.[0, L)\right\}$, where $C^{\infty}[0, L]$ is the collection of infinitely differentiable functions on $[0, L]$ and $R^{5}$ is Euclidean 5 -space.

b. $X=\bar{P}$, the closure of $P$, where the closure is with respect to the norm

$$
\|f\|=\max \left[\max _{0 \leq x \leq L}\left|f_{1}(x)\right|,\left|f_{2}\right|,\left|f_{3}\right|,\left|f_{4}\right|,\left|f_{5}\right|,\left|f_{6}\right|\right] .
$$


c. $D(A)=\left\{f\right.$ in $X: A f$ is in $X, d f_{1}(0) / d x-h_{1} f_{1}(0)=0, d f_{1}(L) / d x+$ $\left.h_{2} f_{1}(L)=0\right\}$.

The existence of a unique solution of problem $(2.8),(2.9)$ belonging to $D(A)$ is demonstrated in the next section.

III. Existence of a unique solution. The main results of this section are that the operator $A$ and its domain $D(A)$ satisfy the hypotheses of the Hille-Yosida theorem, thus yielding the fact that $A$ is the infinitesimal generator of a strongly continuous semigroup, that this semigroup is a compact analytic semigroup, that the operator $B$ is bounded on $X$, that Eq. $(2.8 \mathrm{a})$ is a semilinear evolution equation, and that $F(U)$ satisfies a Lipschitz condition. These results yield the existence of a unique solution of problem $(2.8),(2.9)$ on $D(A)$ which is defined by definition (2.10).

The version of the Hille-Yosida theorem being used is (see [4], [5], [6], [7], or [8] and references therein):

THEOREM. A linear operator $A$ is a generator of a strongly continuous semigroup of contractions if and only if

1. $A$ is closed and $\overline{D(A)}=X$.

2. The resolvent set $p(A)$ of $A$ contains $R^{+}$and for every $z$ in $R^{+}$,

$$
\|R(z ; A)\| \leq 1 / z
$$

where $R(z ; A)=(z I-A)^{-1}$, and $R^{+}$is the positive real axis.

The following lemma establishes that $\overline{D(A)}=X$.

LEMMA 3.1. $\overline{D(A)}=X$.

Proof. $P$ belongs to $D(A)$ which belongs to $X=\bar{P}$, so $\bar{P}$ belongs to $\overline{D(A)}$ which belongs to $X=\bar{P}$. Thus $\overline{D(A)}=X$.

Condition 2 of the Hille-Yosida theorem is established by the following lemma.

Lemma 3.2. The resolvent set $p(A)$ of $A$ contains $R^{+}$and for every $z$ in $R^{+}$, $\|R(z ; A)\| \leq 1 / z$.

Proof. The proof of this lemma follows using arguments much like those in Lemma 3.3 and Theorem 3.4 and is omitted.

Given the results of Lemmas 3.1 and 3.2, the only hypothesis of the Hille-Yosida theorem which needs to be verified is that $A$ is closed. But that $A$ is closed follows from the fact that $(z I-A)^{-1}$ is bounded, so $(z I-A)$ is closed and hence $A$ is closed (see [4], [7], or [8] for details).

By combining Lemmas 3.1 and 3.2 and the preceding remark, the Hille-Yosida theorem can be applied to the operator $A$ of Eq. (2.8) to yield the result that $A$ is the generator of a strongly continuous semigroup $T(t)$ for which

$$
\|T(t)\| \leq 1 \text {. }
$$

However, $T(t)$ satisfies an even stronger condition than being a strongly continuous semigroup, namely $T(t)$ is an analytic semigroup. (See [5], [7], or [8] for a definition.) To verify that the uniformly bounded, strongly continuous semigroup $T(t)$ is analytic, it suffices to show that the following conditions hold

C1. 0 belongs to $p(A)$, the resolvent set of $A$. 
C2. There exists $0<d<\pi / 2$ and $M>0$ such that $p(A)$ contains $S=$ $\{z:|\arg z|<\pi / 2+d\} \cup\{0\}$ and $\|R(z ; A)\| \leq M /|z|$ for all $z$ in $S, z$ not 0 .

See [5], [7], or [8] for proofs that these two conditions are sufficient to establish the analyticity of $T(t)$. These two conditions will now be established.

LemmA 3.3. If $z$ does not belong to $R^{-}$, the negative real axis, then $(z I-A)^{-1}$ exists. Moreover, $z=0$ belongs to $p(A)$.

Proof. Suppose that $z$ is not in $R^{-} \cup\{0\}$. Let $(z I-A) f=g$ where $g$ is in $X$ and $f$ is in $D(A)$. Then $f$ must satisfy the equations

$$
\begin{aligned}
f_{1}^{\prime \prime}-z f_{1} / k & =-g_{1} / k, \\
(1+z) f_{m} & =g_{m} \text { for } m=2, \ldots, 6 .
\end{aligned}
$$

The solution of system (3.3) belonging to $D(A)$ is

$$
\begin{aligned}
f_{1}(x)=[ & I_{1}(x) \int_{0}^{L}\left(a \cosh (a(L-y))+h_{2} \sinh (a(L-y)) g_{1}(y) d y\right. \\
& \left.-E(a) \int_{0}^{x} \sinh (a(x-y)) g_{1}(y) d y\right] /(k a E(a))
\end{aligned}
$$

and

$$
f_{m}=g_{m} /(1+z) \text { for } m=2, \ldots, 6
$$

where

$$
\begin{aligned}
a & =(z / k)^{1 / 2}, \\
I_{1}(x) & =e^{a x}+\left(a-h_{1}\right) e^{-a x} /\left(a+h_{1}\right), \\
I_{2}(x) & =e^{a x}-\left(a-h_{1}\right) e^{-a x} /\left(a+h_{1}\right), \\
E(a) & =a I_{2}(L)+h_{2} I_{1}(L) .
\end{aligned}
$$

The fact that $f$ belongs to $D(A)$ follows from (1) $f$ belongs to $X,(2) A f$ belongs to $X$, and (3) $f_{1}^{\prime}(0)-h_{1} f_{1}(0)=f_{1}^{\prime}(L)+h_{2} f_{1}(L)=0$. Thus, for each $g$ in $X$, there is an $f$ in $D(A)$ such that $(z I-A) f=g$. Thus, $(z I-A)$ is onto. To see that $(z I-A)$ is one-to-one, suppose $(z I-A) f=0$. Then $f_{m}=0$ for $m=2, \ldots, 6$ and $f_{1}^{\prime \prime}=z f_{1} / k$. Thus

$$
\int_{0}^{L} f_{1}^{\prime \prime} \bar{f}_{1} d x=\left[z \int_{0}^{L} f_{1} \bar{f}_{1} d x\right] / k
$$

or, after integrating by parts and using the boundary conditions for $f_{1}$,

$$
-h_{2}\left|f_{1}(L)\right|^{2}-h_{1}\left|f_{1}(0)\right|^{2}-\int_{0}^{L}\left|f_{1}^{\prime}(x)\right|^{2} d x=\left[z \int_{0}^{L}\left|f_{1}(x)\right|^{2} d x\right] / k .
$$

Thus $f_{1}(x)=0$, and $(z I-A)$ is one-to-one. Thus $(z I-A)^{-1}$ maps $X$ to $D(A)$. The fact that $\left\|(z I-A)^{-1}\right\|$ is finite follows from (3.4), (3.5), and an inequality similar to (3.12). 
Suppose $z=0$. Then $(z I-A) f=g$ results in the equations

$$
\begin{aligned}
-k f_{1}^{\prime \prime} & =g_{1}, \\
f_{m} & =g_{m} \text { for } m=2, \ldots, 6 .
\end{aligned}
$$

The solution of Eq. (3.7) which lies in $D(A)$ is

$$
f_{1}(x)=q x+q / h_{1}-\left[\int_{0}^{x}\left\{\int_{0}^{y} g_{1}(s) d s\right\} d y\right] / k
$$

where

$$
q=\left[\int_{0}^{L} g_{1}(y) d y+h_{2} \int_{0}^{L}\left\{\int_{0}^{y} g_{1}(s) d s\right\} d y\right] / d_{1}
$$

and

$$
d_{1}=k\left(1+h_{2} L+h_{2} / h_{1}\right)
$$

Thus,

$$
\left|f_{1}(x)\right| \leq|q|\left(L+1 / h_{1}\right)+\max _{0 \leq y \leq L}\left|g_{1}(y)\right| L^{2} / k
$$

But,

$$
|q| \leq \max _{0 \leq y \leq L}\left|g_{1}(y)\right|\left(L+h_{2} L^{2} / 2\right) / d_{1} .
$$

Thus,

$$
\left|f_{1}(x)\right| \leq \max _{0 \leq y \leq L}\left|g_{1}(y)\right|\left\{L^{2} / k+\left(L+h_{2} L^{2} / 2\right)\left(L+1 / h_{1}\right) / d_{1}\right\},
$$

so,

$$
\left|f_{1}(x)\right| \leq K\|g\|
$$

Thus,

$$
\|f\| \leq K^{*}\|g\|, \quad \text { where } K^{*}=\max [1, K],
$$

and $z=0$ is in $p(A)$.

Now the fact that $T(t)$ is an analytic semigroup will be established.

THEOREM 3.4. $T(t)$ is an analytic semigroup.

Proof. Since the preceding lemma establishes that 0 belongs to $p(A)$ and that $(z I-A)^{-1}$ exists for $z$ not in $R^{-}$, we need only show that condition $\mathrm{C} 2$ holds. Let $d=\pi / 4$ and $z=r e^{i v}$ with $|v|<3 \pi / 4$. Let $g$ be in $X$ and $f$ satisfy $(z I-A) f=g$. Then $f$ is given by Eqs. (3.4) and (3.5) with $a, I_{1}, I_{2}$, and $E$ defined in Eq. (3.6). To obtain the estimate required at this point, it is necessary to rewrite $f_{1}(x)$ as

$$
\begin{aligned}
f_{1}(x)= & {\left[\int_{x}^{L}\left(a G(x, y)+h_{2} H(x, y)\right) g_{1}(y) d y\right.} \\
& \left.\quad+\int_{0}^{x}\left(a G(y, x)+h_{2} H(y, x)\right) g_{1}(y) d y\right] /(k a E(a))
\end{aligned}
$$

where

$$
\begin{aligned}
& G(x, y)=I_{1}(x) \cosh (a(L-y)) \\
& H(x, y)=I_{1}(x) \sinh (a(L-y)) .
\end{aligned}
$$


Since $z$ is complex, $a$ is now complex, so let $a=a_{1}+i a_{2}$. Then from Eq. (3.10)

$$
\begin{aligned}
\left|f_{1}(x)\right| \leq & \max _{0 \leq y \leq L}\left|g_{1}(y)\right| \\
& \times\left\{\int_{x}^{L}\left[|a||G(x, y)|+h_{2}|H(x, y)|\right] d y\right. \\
& \left.+\int_{0}^{x}\left[|a||G(y, x)|+h_{2}|H(y, x)|\right] d y\right\} /[k|a||E(a)|] .
\end{aligned}
$$

Let

$$
I_{3}(x)=\exp \left(a_{1} x\right)+\left|a-h_{1}\right| \exp \left(-a_{1} x\right) /\left|a+h_{1}\right|
$$

and

$$
I_{4}(x)=\exp \left(a_{1} x\right)-\left|a-h_{1}\right| \exp \left(-a_{1} x\right) /\left|a+h_{1}\right|
$$

Then

$$
|G(x, y)| \leq I_{3}(x) \cosh \left(a_{1}(L-y)\right)
$$

and

$$
|H(x, y)| \leq I_{3}(x) \cosh \left(a_{1}(L-y)\right) \text {. }
$$

Thus

$$
\begin{aligned}
\left|f_{1}(x)\right| \leq & \|g\|\left\{I_{3}(x)\left(|a|+h_{2}\right) \int_{x}^{L} \cosh \left(a_{1}(L-y)\right) d y\right. \\
& \left.+\cosh \left(a_{1}(L-x)\right)\left(|a|+h_{2}\right) \int_{0}^{x} I_{3}(y) d y\right\} /[k|a||E(a)|] .
\end{aligned}
$$

Performing the integrations and regrouping results in

$$
\begin{array}{r}
\left|f_{1}(x)\right| \leq\|g\|\left(|a|+h_{2}\right)\left[I_{4}(L)+\left(\left|a-h_{1}\right| /\left|a+h_{1}\right|-1\right) \cosh \left(a_{1}(L-x)\right)\right] \\
\quad /\left[k|a| a_{1}|E(a)|\right] .
\end{array}
$$

Since $k a^{2}=z=r e^{i v}, a=(r / k)^{1 / 2} e^{i v / 2}$ with $-3 \pi / 8<v / 2<3 \pi / 8$. Thus, since $z$ is not zero, $a_{1}=(r / k)^{1 / 2} \cos (v / 2)>0$. Thus

$$
0 \leq\left|a-h_{1}\right| /\left|a+h_{1}\right|<1 \text { and } 0 \leq\left|a-h_{2}\right| /\left|a+h_{2}\right|<1 \text {. }
$$

Moreover, $\cosh \left(a_{1}(L-x)\right)>0$. Hence

$$
\left|f_{1}(x)\right| \leq\|g\|\left(|a|+h_{2}\right) I_{4}(L) /\left[k|a| a_{1}|E(a)|\right] .
$$

Now,

$$
\begin{aligned}
|E(a)| & \geq|| a+h_{2}\left|\exp \left(a_{1} L\right)-\right| a-h_{2}|| a-h_{1}\left|\exp \left(-a_{1} L\right) /\right| a+h_{1}|| \\
& \geq\left|a+h_{2}\right| \exp \left(a_{1} L\right)-\left|a+h_{2}\right|\left|a-h_{1}\right| \exp \left(-a_{1} L\right) /\left|a+h_{1}\right| \\
& =\left|a+h_{2}\right| I_{4}(L) .
\end{aligned}
$$

Also,

and

$$
|a|=\left[a_{1}^{2}+a_{2}^{2}\right]^{1 / 2}<\left[\left(a_{1}+h_{2}\right)^{2}+a_{2}^{2}\right]^{1 / 2}=\left|a+h_{2}\right|
$$

$$
h_{2}<\left[\left(a_{1}+h_{2}\right)^{2}+a_{2}^{2}\right]^{1 / 2}=\left|a+h_{2}\right|
$$


since

$$
h_{2}>0 \text { and } a_{1}>0
$$

Thus

$$
\left|f_{1}(x)\right|<2\|g\|\left|a+h_{2}\right| I_{4}(L) /\left[k|a| a_{1}\left|a+h_{2}\right| I_{4}(L)\right]
$$

or

$$
\left|f_{1}(x)\right|<2\|g\| /\left[k|a| a_{1}\right] .
$$

But $a_{1}=|a| \cos (v / 2)$ and $|z|=k|a|^{2}$. Thus

$$
\left|f_{1}(x)\right|<2\|g\| /\left[k|a|^{2} \cos (v / 2)\right]=M_{1}\|g\| /|z|
$$

where

$$
M_{1}=2 / \cos (3 \pi / 8)
$$

From Eq. (3.5)

$$
\left|f_{i}\right|=\left|g_{i}\right| /|1+z| \text { for } i=2, \ldots, 6
$$

But

$$
|1+z|^{2}=(1+r \cos v)^{2}+r^{2} \sin ^{2} v=1+2 r \cos v+r^{2} .
$$

Also, $|v|<3 \pi / 4$, so $-2^{1 / 2} / 2<\cos v<1$. Thus $1+r^{2}-2^{1 / 2} r<1+2 r \cos v+r^{2}=$ $|1+z|^{2}$. But $0 \leq\left(r-2^{1 / 2}\right)^{2}=r^{2}-2^{3 / 2} r+2$, so $r^{2} \leq 2\left(r^{2}-2^{1 / 2} r+1\right)<2|1+z|^{2}$. Thus,

$$
|z|^{2}<2|1+z|^{2}
$$

or,

$$
|z|<2^{1 / 2}|1+z|
$$

Thus,

$$
\left|f_{1}\right|<2^{1 / 2}\left|g_{i}\right| /|z|<2^{1 / 2}\|g\| /|z| \text { for } i=2, \ldots, 6 \text {. }
$$

Since $2^{1 / 2}<2 / \cos (3 \pi / 8)$,

$$
\|f\|<M_{1}\|g\| /|z| .
$$

Therefore $\|R(z ; A)\|<M_{1} /|z|$ and condition $\mathrm{C} 2$ is satisfied.

There is one more property of $T(t)$ which is established in the following lemma.

LEMMA 3.5. $T(t)$ is a compact semigroup.

Proof. The proof follows exactly as the proof of Lemma 8.2.1 of [8]. Since $T(t)$ is analytic, it is continuous in the uniform operator topology for $t>0$. Also, for each $z$ in $p(A), R(z ; A)$ maps $X$ into $D(A)$ such that bounded sets in $X$ are mapped into bounded sets in $D(A)$ which contain entries which also have a uniform bound on their first derivative. Thus it follows from the Arzela-Ascoli theorem that $R(z ; A)$ is a compact operator. It follows from Theorem 2.3.3 of [8] that $T(t)$ is a compact semigroup for $t \geq 0$.

The next step in demonstrating the existence of a solution of problem (2.8), (2.9) is to show that the operator $B$ of Eq. (2.8) is a bounded operator from $X$ into $X$. 
LEMMA 3.6. Let

$$
\begin{aligned}
& M_{2}=\max \left\{(|m| L+|b|)\left(\left|B_{21}\right|+\left|B_{22}\right|+\left|B_{23}\right|\right),\right. \\
& \left.\left|B_{21}\right|+\left|B_{22}\right|+\left|B_{23}\right|+1,\left|B_{32}\right|+\left|B_{33}\right|+1\right\} .
\end{aligned}
$$

Then $\|B U\| \leq M_{2}\|U\|$ for each $U$ in $X$, so $B$ is bounded on $X$.

Proof. Follows directly from definition of $B, M_{2}$ and the norm.

This lemma and results concerning bounded perturbations of generators of semigroups (see [4], [6], [7], or [8]) yield the following corollary.

Corollary 3.7. If $A$ and $B$ are defined by Eq. (2.8), then $A+B$ is the infinitesimal generator of a compact analytic semigroup $S(t)$ which satisfies

$$
S(t) \leq M_{1} \exp [\|B\| t] .
$$

Proof. Follows directly from Lemma 3.6 and theorems concerning bounded perturbations of compact analytic semigroups.

The $F(U)$ term of Eq. (2.8) which causes the equation to be semilinear will now be considered. Various existence theorems appear in [6], [7], [8], and [9]. The one to be used here is from [8] or [9]. A classical solution of problem $(2.8),(2.9)$ on $[0, T)$ is a function $U(t)$ in $X$ which satisfies

1. $U(t)$ is continuous on $[0, T)$;

2. $U(t)$ is continuously differentiable on $(0, T)$;

3. $U(t)$ belongs to $D(A)$ for $0<t<T$;

4. $U(t)$ satisfies Eqs. $(2.8)$ on $(0, T)$ and (2.9).

The following lemma establishes that $F(U)$ defined in Eq. (2.8) satisfies the hypotheses of the existence and uniqueness theorem of [8, Theorem 8.2.2] or reference [9, Theorem 5.2].

Lemma 3.8. Let $Q=\left\{U\right.$ in $\left.X:-.9 N_{2}<u_{6}\right\}$, then $F(U)$ defined in Eq. (2.8) satisfies

$$
\left\|F(U)-F\left(U^{*}\right)\right\| \leq M_{3}\left\|U-U^{*}\right\|
$$

for every $U, U^{*}$ in $Q$, where $M_{3}=\max \left[(|m| L+|b|) C_{1}, C_{1}, 100\left|N_{1}\right| / N_{2}\right]$ and $C_{1}=\left|B_{21}\right|\left(h_{1} L+1\right) h_{2} R_{2} /\left(h_{1}+h_{2}+h_{1} h_{2} L\right)$.

Procf. From Eqs. (2.8), (2.7), and (2.5a),

$$
F(U)-F\left(U^{*}\right)=\left[\begin{array}{c}
\varphi(x) B_{21}\left(B_{F 2} u_{2}-B_{F 2} u_{2}^{*}\right) \\
B_{21}\left(B_{F 2} u_{2}-B_{F 2} u_{2}^{*}\right) \\
0 \\
0 \\
0 \\
N_{1} u_{6} /\left(N_{2}+u_{6}\right)-N_{1} u_{6}^{*} /\left(N_{2}+u_{6}^{*}\right)
\end{array}\right] .
$$

Note that

$$
\left|\varphi(x) B_{21}\left(B_{F 2} u_{2}-B_{F 2} u_{2}^{*}\right)\right| \leq(|m| L+|b|) C_{1}\left|u_{2}-u_{2}^{*}\right|
$$

and

$$
\left|B_{21}\left(B_{F 2} u_{2}-B_{F 2} u_{2}^{*}\right)\right| \leq C_{1}\left|u_{2}-u_{2}^{*}\right|
$$


Also, if $U$ and $U^{*}$ are in $Q$, then

$$
\begin{aligned}
\left|N_{1} u_{6} /\left(N_{2}+u_{6}\right)-N_{1} u_{6}^{*} /\left(N_{2}+u_{6}^{*}\right)\right| & =\left|N_{1}\right| N_{2}\left|u_{6}-u_{6}^{*}\right| /\left|\left(N_{2}+u_{6}^{*}\right)\left(N_{2}+u_{6}\right)\right| \\
& <100\left|N_{1}\right|\left|u_{6}-u_{6}^{*}\right| / N_{2} .
\end{aligned}
$$

Thus,

$$
\left\|F(U)-F\left(U^{*}\right)\right\| \leq M_{3}\left\|U-U^{*}\right\| .
$$

Corollary 3.9. For every $U_{0}$ in $Q$ there is a $t_{0}>0$ such that problem (2.8), (2.9) has a unique classical solution $U(t)$ on $\left[0, t_{0}\right]$.

Proof. From Corollary 3.7 and Lemma 3.8, problem (2.8), (2.9) satisfies the hypotheses of Theorems 2.1 and 5.2 of [9]. Thus the problem has a unique classical solution on $\left[0, t_{0}\right]$ for $t_{0}>0$.

IV. Finite-dimensional approximations. Approximate solutions of problem (2.8), (2.9) can be obtained by replacing the partial derivatives in operator $A$ with finite differences. If the solution is sought at only a finite number of $x$ locations between 0 and $L$, then the approximation to Eq. $(2.8)$ is a system of ordinary differential equations. Let $n$ be a fixed integer and let

$$
\begin{aligned}
d_{n} & =L /(n-1), \\
x_{i} & =(i-1) d_{n} \text { for } i=1,2, \ldots, n .
\end{aligned}
$$

Let $N=n+5$. Then a finite-dimensional space $X^{N}$ is defined by

$$
X^{N}=\left\{f_{N}: f_{N}=\left[\begin{array}{c}
f_{N}^{(1)} \\
\vdots \\
f_{N}^{(n)} \\
f_{N}^{(n+1)} \\
\vdots \\
f_{N}^{(n+5)}
\end{array}\right]\right\}
$$

and the projection $P_{N}$, from $X$ to $X^{N}$ is defined by

$$
P_{N} f=\left[\begin{array}{c}
f_{1}\left(x_{1}\right) \\
\vdots \\
f_{1}\left(x_{n}\right) \\
f_{2} \\
\vdots \\
f_{6}
\end{array}\right]
$$


for each $f$ in $X$. The operator $A_{N}$ is defined on $X^{N}$ by

$$
A_{N} f_{N}=\left[\begin{array}{c}
2 k\left(f_{N}^{(2)}-\left(1+d_{n} h_{1}\right) f_{N}^{(1)}\right) / d_{n}^{2} \\
k\left(f_{N}^{(3)}-2 f_{N}^{(2)}+f_{N}^{(1)}\right) / d_{n}^{2} \\
\vdots \\
k\left(f_{N}^{(n)}-2 f_{N}^{(n-1)}+f_{N}^{(n-2)}\right) / d_{n}^{2} \\
2 k\left(f_{N}^{(n-1)}-\left(1+d_{n} h_{2}\right) f_{N}^{(n)}\right) / d_{n}^{2} \\
-f_{N}^{(n+1))} \\
\vdots \\
-f_{N}^{(n+5)}
\end{array}\right] .
$$

Note that $X^{N}$ is $N=n+5$-dimensional Euclidean space and is a Banach space with the norm $\left\|f_{N}\right\|_{N}=\max _{1 \leq i \leq N}\left|f_{N}^{(i)}\right|$. The following lemma permits application of the Hille-Yosida theorem to the bounded operators $A_{N}$ on the finite-dimensional Banach Spaces, $X^{N}$. The consequence of this result is stated in the corollary.

LemMA 4.1. If $z>0,\left(z I-A_{N}\right)^{-1}$ exists. Moreover, if

$$
\left(z I-A_{N}\right) f_{N}=g_{N}
$$

then

$$
\left\|f_{N}\right\|_{N} \leq\left\|g_{N}\right\|_{N} / z
$$

Thus

$$
\left\|\left(z I-A_{N}\right)^{-1}\right\| \leq 1 / z .
$$

Proof. We can partition $A_{N}$ as

$$
A_{N}=\left[\begin{array}{cc}
A_{N}^{*} & 0 \\
0 & -I
\end{array}\right] \text {. }
$$

For $z>0$, the matrix $z I-A_{N}^{*}$ is strictly diagonally dominant and hence by the Gerschgorin circle theorem it is nonsingular. Also, $z I+I$ is nonsingular, so $z I-A_{N}$ is nonsingular and we can solve $\left(z I-A_{N}\right) f_{N}=g_{N}$.

Since $\left\|f_{N}\right\|_{N}=\max _{0 \leq i \leq N}\left|f_{N}^{(i)}\right|$, there is an $s$ such that

$$
\left\|f_{N}\right\|_{N}=\left|f_{N}^{(s)}\right| \text {. }
$$

If $s=1$ then

$$
\left[z+2 k\left(1+d_{n} h_{1}\right) / d_{n}^{2}\right] f_{N}^{(1)}=g_{N}^{(1)}+2 k f_{N}^{(2)} / d_{n}^{2}
$$

or

$$
\left[z+2 k\left(1+d_{n} h_{1}\right) / d_{n}^{2}\right]\left|f_{N}^{(1)}\right| \leq\left\|g_{N}\right\|+2 k\left\|f_{N}\right\| / d_{n}^{2}
$$

or

$$
\left[z+2 k\left(1+d_{n} h_{1}\right) / d_{n}^{2}\right]\left\|f_{N}\right\| \leq\left\|g_{N}\right\|+2 k\left\|f_{N}\right\| / d_{n}^{2} .
$$

Thus,

$$
z\left\|f_{N}\right\| \leq\left\|g_{N}\right\|
$$


If $2 \leq s \leq n-1$, then

$$
\left[z+2 k / d_{n}^{2}\right] f_{N}^{(s)}=g_{N}^{(s)}+k\left[f_{N}^{(s-1)}+f_{N}^{(s+1)}\right] / d_{n}^{2}
$$

so

$$
\left[z+2 k / d_{n}^{2}\right]\left|f_{N}^{(s)}\right| \leq\left\|g_{N}\right\|+2 k\left\|f_{N}\right\| / d_{n}^{2}
$$

or

$$
\left[z+2 k / d_{n}^{2}\right]\left\|f_{N}\right\| \leq\left\|g_{N}\right\|+2 k\left\|f_{N}\right\| / d_{n}^{2}
$$

Thus,

$$
z\left\|f_{N}\right\| \leq\left\|g_{N}\right\| .
$$

If $s=n$, the argument and conclusion is the same as for the case $s=1$. If $s=n+m$ for $m=1, \ldots, 5$, then

$$
[z+1] f_{N}^{(s)}=g_{N}^{(s)}
$$

so

$$
z\left\|f_{N}\right\| \leq\left\|g_{N}\right\| .
$$

Thus, in every case $z\left\|f_{N}\right\| \leq\left\|g_{N}\right\|$, and the lemma is proved.

Corollary 4.2. $A_{N}$ is the generator of $T_{N}(t)=\exp \left(A_{N} t\right)$ where $\left\|T_{N}\right\| \leq 1$.

Now that $A_{N}$ is seen to generate a strongly continuous semigroup (in fact, a group) of contractions, Lemmas 4.3 and 4.4 permit the application of the approximation theory of Trotter and Kato (see [8]). The consequence of this theorem is stated in the two corollaries following Lemma 4.4.

LEMMA 4.3.

$$
\begin{aligned}
\left\|P_{N} f\right\|_{N} \leq\|f\|_{X} & \text { for every } f \text { in } X, \\
\lim _{N \rightarrow \infty}\left\|P_{N} f\right\|_{N}=\|f\|_{X} & \text { for every } f \text { in } X .
\end{aligned}
$$

Proof.

$$
\begin{aligned}
\left\|P_{N} f\right\|_{N} & =\max \left[\max _{1 \leq i \leq n}\left|f\left(x_{i}\right)\right|,\left|f_{2}\right|, \ldots,\left|f_{6}\right|\right] \\
& \leq \max \left[\max _{0 \leq x \leq L}|f(x)|,\left|f_{2}\right|, \ldots,\left|f_{6}\right|\right]=\|f\|_{X} .
\end{aligned}
$$

Thus $\lim _{N \rightarrow \infty}\left\|P_{N} f\right\| \leq\|f\|_{X}$. But, since $|f|$ is continuous, there is an $x_{0}$ in $[0, L]$ such that $\max \left[\left|f\left(x_{0}\right)\right|,\left|f_{2}\right|, \ldots,\left|f_{6}\right|\right]=\|f\|_{X}$. Also, because $f$ is continuous, if $\varepsilon>$ 0 is given, for $N$ sufficiently large there is an $x_{N}^{(i)}$ for which $\left|f\left(x_{0}\right)\right|-\varepsilon<\left|f\left(x_{N}^{(i)}\right)\right|$. But then

$$
\begin{aligned}
\left\|P_{N} f\right\| & \geq \max \left[\mid f\left(x_{N}^{(i)}|,| f_{2}|, \ldots,| f_{6} \mid\right]\right. \\
& >\max \left[\left|f\left(x_{0}\right)\right|-\varepsilon,\left|f_{2}\right|, \ldots,\left|f_{6}\right|\right] \geq\|f\|_{X}-\varepsilon .
\end{aligned}
$$

Thus, because $\varepsilon$ is arbitrarily small, the lemma is proved. 
LEMMA 4.4.

$$
\begin{aligned}
P_{N} f \text { is in } D\left(A_{N}\right) & =X^{N} \quad \text { for every } f \text { in } D(A) . \\
\lim _{N \rightarrow \infty}\left\|A_{N} P_{N} f-P_{N} A f\right\|_{N} & =0 \quad \text { for every } f \text { in } D(A) .
\end{aligned}
$$

Proof. The fact that $P_{N} f$ is in $D\left(A_{N}\right)$ for every $f$ in $D(A)$ is a straightforward consequence of the definitions of $P_{N}$ and $A_{N}$. Moreover,

$$
A_{N} P_{N} f-P_{N} A f=\left[\begin{array}{c}
2 k\left[f\left(x_{2}\right)-\left(1+d_{n} h_{1}\right) f\left(x_{1}\right)\right] / d_{n}^{2}-k f^{\prime \prime}\left(x_{1}\right) \\
k\left[f\left(x_{3}\right)-2 f\left(x_{2}\right)+f\left(x_{1}\right)\right] / d_{n}^{2}-k f^{\prime \prime}\left(x_{2}\right) \\
\vdots \\
k\left[f\left(x_{n}\right)-2 f\left(x_{n-1}\right)+f\left(x_{n+1}\right)\right] / d_{n}^{2}-k f^{\prime \prime}\left(x_{n-1}\right) \\
2 k\left[f\left(x_{n-1}\right)-\left(1+d_{n} h_{2}\right) f\left(x_{n}\right)\right] / d_{n}^{2}-k f^{\prime \prime}\left(x_{n}\right) \\
0 \\
0 \\
0 \\
0 \\
0
\end{array}\right] .
$$

Let $\varepsilon>0$ be given. By Taylor's theorem, if $d_{n}$ is sufficiently small (i.e., if $n$ is sufficiently large), the second through the $(n-1)$ st term of $A_{N} P_{N} f-P_{N} A f$ will be less than $\varepsilon$ in absolute value. For the first term we use the fact that $f^{\prime}\left(x_{1}\right)=h_{1} f\left(x_{1}\right)$, since $f$ belongs to $D(A)$. Thus, by Taylor's theorem

$$
f\left(x_{2}\right)=f\left(x_{1}\right)+h_{1} f\left(x_{1}\right) d_{n}+f^{\prime \prime}\left(x^{*}\right) d_{n}^{2} / 2
$$

or

$$
2\left[f\left(x_{2}\right)-\left(1+d_{n} h_{1}\right) f\left(x_{1}\right)\right] / d_{n}^{2}=f^{\prime \prime}\left(x^{*}\right)
$$

where $x_{1}<x^{*}<x_{2}$. Again, if $N$ is sufficiently large, the first term of $A_{N} P_{N} f-$ $P_{N} A f$ is less than $\varepsilon$ in absolute value. The $n$th term is handled in the same way as the first term. Thus the lemma is proved.

Corollary 4.5. $\lim _{N \rightarrow \infty}\left\|R\left(z, A_{N}\right) P_{N} f-P_{N} R(z, A) f\right\|_{N}=0$ for every $f$ in $X$, where $R\left(z, A_{N}\right)=\left(z I-A_{N}\right)^{-1}$.

Proof. This follows from Lemmas 3.2, 4.1, 4.3, 4.4, and the arguments in [4, p. 230].

Corollary 4.6. $\lim _{N \rightarrow \infty}\left\|T_{N}(t) P_{N} f-P_{N} T(t) f\right\|_{N}=0$, for every $f$ in $X, t \geq 0$, with the convergence uniform on compact subsets of $[0, \infty)$.

Proof. Follows from Theorem 5.2 of [10]. See also [8] and [4]. 
The approximation of problem (2.8) on $X_{N}$ requires the definition of an operator corresponding to $B$. Thus $B_{N}$ is defined by

$$
B_{N} f_{N}=\left[\begin{array}{c}
\left(m x_{1}+b\right)\left[B_{21} f_{N}^{(n)}+B_{22} f_{N}^{(n+1)}+\cdots+B_{26} f_{N}^{(n+5)}\right] \\
\vdots \\
\left(m x_{n}+b\right)\left[B_{21} f_{N}^{(n)}+B_{22} f_{N}^{(n+1)}+\cdots+B_{26} f_{N}^{(n+5)}\right] \\
B_{21} f_{N}^{(n)}+\left(B_{22}+1\right) f_{N}^{(n+1)}+\cdots+B_{26} f_{N}^{(n+5)} \\
\left.B_{32} f_{N}^{(n+1)}+\cdots+B_{36}+1\right) f_{N}^{(n+5)} \\
\vdots \\
B_{62} f_{N}^{(n+1)}+\cdots+\left(B_{66}+1\right) f_{N}^{(n+5)}
\end{array}\right]
$$

for each $f_{N}$ in $X^{N}$. An immediate consequence of the fact that $B_{N} P_{N} f=P_{N} B f$ is the following lemma.

Lemma 4.7. $\lim _{N \rightarrow \infty}\left\|\exp \left(t\left(A_{N}+B_{N}\right)\right) P_{N} f-P_{N} S(t) f\right\|_{N}=0$ for every $f$ in $X$ and $t \geq 0$, uniformly on compact subsets of $[0, \infty)$, where $S(t)$ is referenced in Corollary 3.7.

Proof. Follows directly from [4, Theorem 6.3].

Corollary 4.8. If, for $U_{0}$ not equal to $0, U_{N}$ is the solution of

and $U$ is the solution of

$$
\begin{aligned}
d U_{N} / d t & =A_{N} U_{N}+B_{N} U_{N}, \\
U_{N}(0) & =P_{N} U_{0},
\end{aligned}
$$

$$
\begin{aligned}
U_{t} & =A U+B U, \\
U(0) & =U_{0},
\end{aligned}
$$

then

$$
\lim _{N \rightarrow \infty}\left\|U_{N}(t)-P_{N} U(t)\right\|_{N}=0
$$

Proof. Since $A_{N}$ and $B_{N}$ are both bounded operators mapping $X_{N}$ to $X_{N}$, $U_{N}(t)=\exp \left(t\left(A_{N}+B_{N}\right)\right) P_{N} U_{0}$. As a result of Corollary 3.7, $U(t)=S(t) U_{0}$, (where $S(t)$ is defined in Corollary 3.7). Thus we may use Lemma 4.7 with $f$ replaced with $U_{0}$ to see the lemma is proved.

Now suppose the operator $F_{N}$ is defined on

$$
Q_{N}=\left\{f_{N} \text { in } X_{N}:-.9 N_{2}<f_{N}^{(n+5)}\right\}
$$

by

$$
F_{N}\left[f_{N}\right]=\left[\begin{array}{c}
\left(m x_{1}+b\right) B_{21}\left(B_{F 1}+B_{F 2} f_{N}^{(n+1)}\right) \\
\vdots \\
\left(m x_{n}+b\right) B_{21}\left(B_{F 1}+B_{F 2} f_{N}^{(n+1)}\right) \\
B_{21}\left(B_{F 1}+B_{F 2} f_{N}^{(n+1)}\right) \\
\vdots \\
N_{1} f_{N}^{(n+5)} /\left(N_{2}+f_{N}^{(n+5)}\right)
\end{array}\right]
$$

Note that $F_{N}\left[P_{N} f\right]=P_{N} F[f]$. Moreover, the next lemma indicates that $F_{N}$ satisfies a result similar to Lemma 3.8. 
Lemma 4.9. $F_{N}$ defined by (4.6) satisfies

$$
\left\|F_{N}\left(f_{N}\right)-F_{N}\left(f_{N}^{*}\right)\right\| \leq M_{4}\left\|f_{N}-f_{N}^{*}\right\|
$$

for every $f_{N}, f_{N}^{*}$ in $X_{N}$, where $M_{4}$ is constant.

Proof. Because of the definition of $F_{N}$, the proof is basically the same as the proof of Lemma 3.8.

Because $S_{N}(t)=\exp \left(t\left(A_{N}+B_{N}\right)\right)$ is bounded and has a finite-dimensional range, $S_{N}(t)$ is a compact analytic semigroup. Thus we have the following lemma.

Lemma 4.10. For every $U_{0}$, the approximate problem

$$
\begin{gathered}
d U_{N} / d t=A_{N} U_{N}+B_{N} U_{N}+F_{N}\left[U_{N}\right] \\
U_{N}(0)=P_{N} U_{0}
\end{gathered}
$$

has a unique classical solution $U_{N}(t)$ on $\left[0, t_{N}^{*}\right]$ for $t_{N}^{*}$ sufficiently small.

Proof. The proof follows from Lemma 4.9 and the fact that $S_{N}(t)$ is a compact analytic semigroup.

We can now establish that the solution which Lemma 4.10 guarantees converges to the solution which Corollary 3.9 guarantees.

LEMMA 4.11. If $U(t)$ is the unique classical solution of problem (2.8), (2.9) and $U_{N}(t)$ is the unique classical solution of problem (4.7), (4.8), then

$$
\lim _{N \rightarrow \infty}\left\|P_{N} U(t)-U_{N}(t)\right\|_{N}=0
$$

Proof. Since classical solutions are mild solutions, $U(t)$ and $U_{N}(t)$ satisfy the respective integral equations

$$
\begin{gathered}
U(t)=S(t) U_{0}+\int_{0}^{t} S(t-s) F(U(s)) d s, \\
U_{N}(t)=S_{N}(t) P_{N} U_{0}+\int_{0}^{t} S_{N}(t-s) F_{N}\left(U_{N}(s)\right) d s,
\end{gathered}
$$

where $S_{N}(t)=\exp \left(t\left(A_{N}+B_{N}\right)\right)$. Let $\varepsilon>0$ be given and let $t$ satisfy $0 \leq t \leq t_{0}$, where $t_{0}$ is given in Corollary 3.9. Now choose $J>0$ so that

$$
\left\|P_{N} S(t) U_{0}-S_{N}(t) P_{N} U_{0}\right\|_{N}<\varepsilon / 4
$$

and

$$
\left\|P_{N} S(t-s) F(U(s))-S_{N}(t-s) P_{N} F(U(s))\right\|_{N}<\varepsilon / 4
$$

for all $N \geq J$ and all $s, t$ in $\left[0, t_{0}\right]$. Let $N=J, t_{0}^{*}=\min \left[t_{0}, t_{N}^{*}\right]$, and $0 \leq t \leq t_{0}^{*}$. 
Then

$$
\begin{aligned}
\left\|P_{N} U(t)-U_{N}(t)\right\|_{N} \leq & \left\|P_{N} S(t) U_{0}-S_{N}(t) P_{N} U_{0}\right\|_{N} \\
& +\left\|\int_{0}^{t}\left[P_{N} S(t-s) F(U(s))-S_{N}(t-s) F_{N}\left(U_{N}(s)\right)\right] d s\right\|_{N} \\
\leq & \left\|P_{N} S(t) U_{0}-S_{N}(t) P_{N} U_{0}\right\|_{N} \\
& +\int_{0}^{t}\left[\left\|P_{N} S(t-s) F(U(s))-S_{N}(t-s) P_{N} F(U(s))\right\|_{N}\right. \\
& \quad+\left\|S_{N}(t-s) P_{N} F(U(s))-S_{N}(t-s) F_{N}\left(P_{N} U(s)\right)\right\|_{N} \\
& \left.\quad+\left\|S_{N}(t-s) F_{N}\left(P_{N} U(s)\right)-S_{N}(t-s) F_{N}\left(U_{N}(s)\right)\right\|_{N}\right] d s \\
< & \varepsilon / 4+t \varepsilon / 4+M_{4} e^{w t} \int_{0}^{t}\left\|P_{N} U(s)-U_{N}(s)\right\|_{N},
\end{aligned}
$$

where $w=\left\|A_{N}\right\|+\left\|B_{N}\right\|$. The second term under the integral sign before the last inequality vanishes because of the definition of $F_{N}$. An application of Gronwall's inequality then yields that $\left\|P_{N} U(t)-U_{N}(t)\right\|_{N} \rightarrow 0$ as $N \rightarrow \infty$.

As indicated in the Introduction, numerical results are presented in the next section.

V. Numerical results. Experimental measurements of concentrations of dibromomethane in the blood of laboratory rats are given in [1] for four different concentrations of exposure on the skin. The measured concentrations were in milligrams per milliliter, while the skin exposure concentrations were at 500 parts per million, 1000 PPM, 5000 PPM and 10,000 PPM. The concentration on the skin was initially zero and then was increased rapidly to the constant peak concentration.

In Fig. 2 the circles denote the experimental data and the solid curves are the approximate solution of the system of differential equations corresponding to Eqs. (4.7), (4.8). Because Eq. (4.7) is a nonlinear system of ordinary differential equations, numerical solutions were obtained using the IMSL routine DGEAR. From Fig. 2 we see that our model is extremely accurate in terms of determining the arterial blood concentrations.

To obtain a quantitative measure of how well the theoretical predictions agree with the experimental data, the following deviation sum of squares is used:

$$
d=\sum_{i=1}^{4} \sum_{j=1}^{5}\left[\left[O_{i}\left(t_{j}\right)-P_{i}\left(t_{j}\right)\right] / w_{i}\right]^{2}
$$

where the $O_{i}\left(t_{j}\right)$ denotes the $j$ th observation for experiment $i$ and the $P_{i}\left(t_{j}\right)$ denotes the prediction at time $t_{j}$ for experiment $i$. The four experiments correspond to the four concentrations of exposure listed previously in this section. The weights are included because the data are of the order of 1 for experiment 1 , while they are of the order of 2 for the second experiment, are of the order of 20 for the third experiment, and of the order of 150 for the fourth experiment. For the ordinary differential equation model, the value of $d$ is 0.629 , while for the model of this paper, $d$ is 0.549 . 


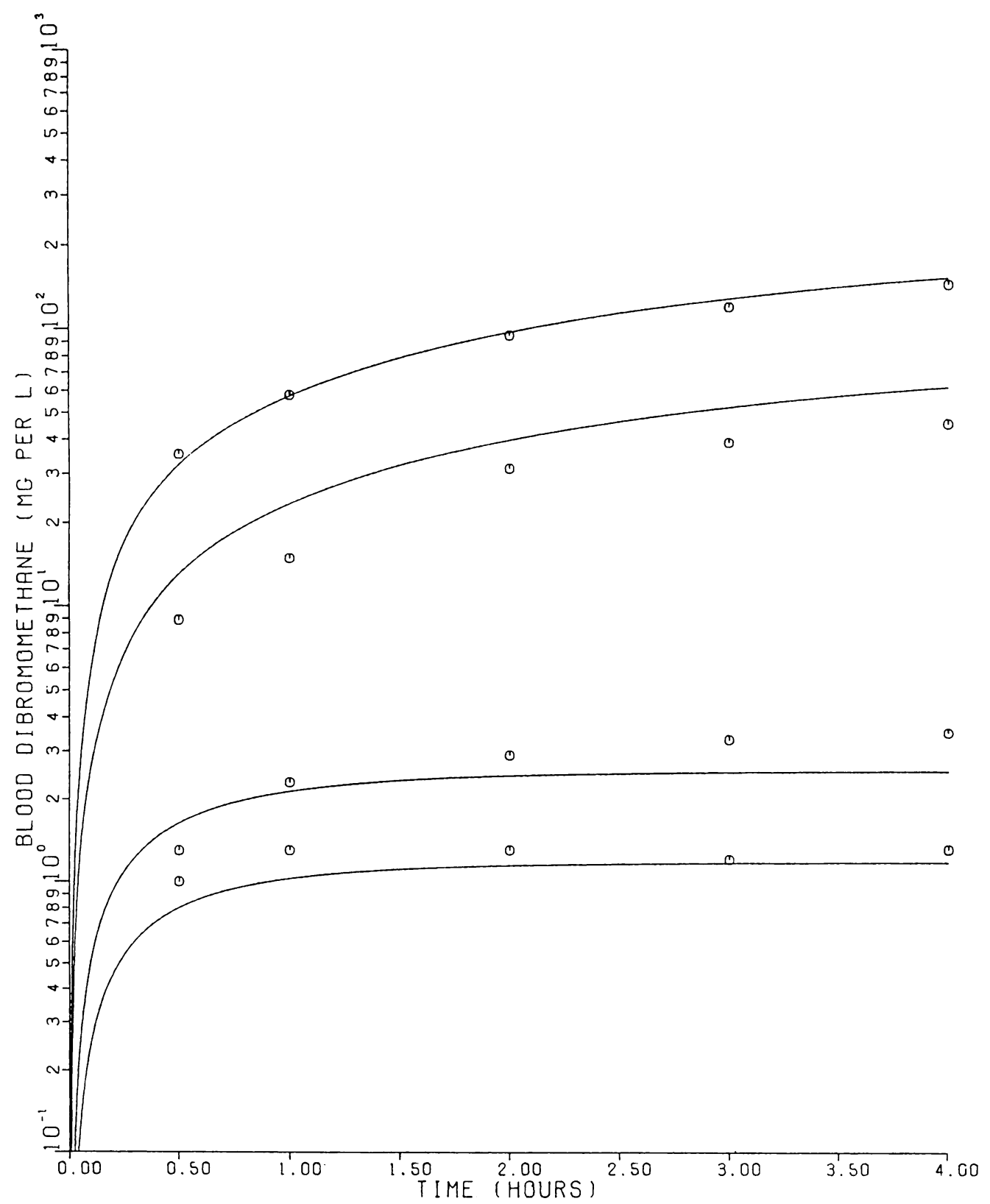

Fig. 2. Comparison of numerical solution with experimental data.

\section{REFERENCES}

[1] J. N. McDougal, G. W. Jepson, H. J. Clewell III, and M. E. Andersen, Dermal absorption of dihalomethane vapors, Toxicology and Applied Pharmacology 79, 150-158 (1985)

[2] J. N. McDougal, G. W. Jepson, H. J. Clewell III, M. G. MacNaughton, and M. E. Andersen, $A$ physiological pharmacokinetic model for dermal absorption of vapors in the rat, Toxicology and Applied Pharmacology 85, 286-294 (1986) 
[3] H. T. Banks and K. Kunisch, An Approximation theory for nonlinear partial differential equations with applications to identification and control, SIAM J. Control Optim. 20, 815-849 (1982)

[4] A. Belleni-Morante, Applied Semigroups and Evolution Equations, Clarendon Press, Oxford, 1980

[5] H. O. Fattorini, The Cauchy Problem, Addison-Wesley, Reading, Massachusetts, 1983

[6] T. Kato, Perturbation Theory for Linear Operators, Springer-Verlag, New York, 1966

[7] R. H. Martin, Jr., Nonlinear Operators and Differential Equations in Banach Spaces, John Wiley \& Sons, New York, 1976

[8] A. Pazy, Semigroups of Linear Operators and Applications to Partial Differential Equations, Springer Verlag, New York, 1983

[9] A. Pazy, A class of semi-linear equations of evolution, Israel J. Math. 20, 23-36 (1975)

[10] H. F. Trotter, Approximation of semi-groups of operators, Pacific J. Math. 8, 887-919 (1959)

[11] D. W. Quinn, A distributed parameter identification problem which arises in pharmacokinetics, Proceedings of the University of Arkansas Eighth Annual Lecture Series in the Mathematical Sciences, 1984 